\title{
Analysis Cloud Running Sensor Data Analysis Programs on a Cloud Computing Infrastructure
}

\author{
Jan Sipke van der Veen ${ }^{1}$, Bram van der Waaij ${ }^{1}$, Matthijs Vonder $^{1}$, Marc de Jonge ${ }^{1}$, Elena Lazovik ${ }^{1}$ \\ and Robert J. Meijer ${ }^{1,2}$ \\ ${ }^{1}$ TNO, Groningen, The Netherlands \\ ${ }^{2}$ University of Amsterdam, Amsterdam, The Netherlands \\ \{jan_sipke.vanderveen, bram.vanderwaaij, matthijs.vonder, marc.dejonge, elena.lazovik\}@tno.nl, rmeijer@science.uva.nl
}

Keywords: $\quad$ Data Analysis, Data Science, Sensor Data, Cloud Computing.

Abstract: $\quad$ Sensors have been used for many years to gather information about their environment. The number of sensors connected to the internet is increasing, which has led to a growing demand of data transport and storage capacity. In addition, evermore emphasis is put on processing the data to detect anomalous situations and to identify trends. This paper presents a sensor data analysis platform that executes statistical analysis programs on a cloud computing infrastructure. Compared to existing batch and stream processing platforms, it adds the notion of simulated time, i.e. time that differs from the actual, current time. Moreover, it adds the ability to dynamically schedule the analysis programs based on a single timestamp, recurring schedule, or on the sensor data itself.

\section{INTRODUCTION}

Sensors have been used for many years to gather information about both physical and virtual environments. Typical applications of these sensors include prediction of the weather based on the current conditions (Blackwell, 2005), adjusting the number of virtual machines of a webservice based on its measured quality of service (Rao et al., 2011), and monitoring physical structures to detect anomalies (Pyayt et al., 2011).

Since sensors are becoming cheaper and simpler to use, the number of sensors connected to the internet has grown rapidly over the years. This has led to a growing demand of sensor data transport and storage capacity (Sheng et al., 2006), but also on systems that make sense of the data and decide on useful actions. The field of autonomic computing (IBM Corporation, 2001) provides a number of steps that such systems should have, see Figure 1. The process starts with a sensor that measures the environment. The Monitor, Analyze, Plan, Execute (MAPE) loop then monitors the output of the sensor, analyzes the measured values to detect problems, plans on a set of actions to remedy the problem, and executes the selected actions. The process ends with an effector to influence the environment. This paper focuses on the second step in the MAPE loop, the analysis of the sensor data.

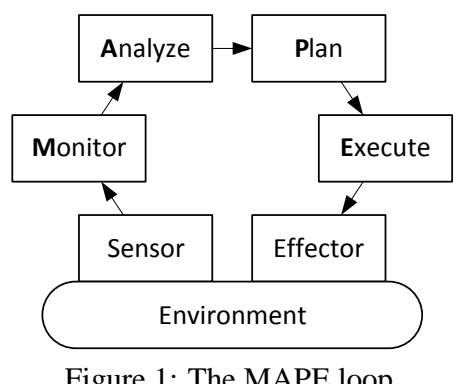

Figure 1: The MAPE loop.

A distinction can be made between general statistical analysis and large scale scientific models to analyse sensor data. Scientific models are typically developed by experts in the field and are executed on high performance computer systems such as supercomputers or grid computing infrastructures. The running time of these models may range from minutes to days or even months. Because of these relatively long running times, it is feasible to use a virtual machine (VM) for each model.

Statistical analysis programs are typically executed on a single computer. They are used to gain a first insight into the sensor data and, if necessary, may lead to further analysis. The running time of these programs is typically in the order of milliseconds to seconds. Because of these relatively short running times and possibly large number of concur- 
rent analysis programs, it is not feasible to use a VM for each program. Therefore, the need arises for a finer-grained distributed platform for the execution of statistical analysis programs.

Processing of data can be performed in batches and in continuous streams. In batch processing, input data is aggregated into a single batch that will all be analysed at one time. Typically, the output of a batch is only visible at the end of the run. An example of a batch process is a retailer taking all sales data of the past week and then calculating sales performance. If the start of the week contains anomalous data, the retailer must wait until the end of the week for the output of the batch process. Historical analysis of sensor data, e.g. calculating the standard deviation for the sensor values of the previous day, fits perfectly into the category of batch processing.

In stream processing, input data is analysed as it arrives and (partial) output is available immediately. This means that one can act on the data as soon as it arrives. However, this may lead to skewed results as the analysis is never actually finished. In the case of the retailer, comparing partial results from one country to the next is difficult because of possibly different time zones and business hours. To be able to quickly respond to anomalous situations, the analysis of current sensor data is performed soon after the data arrives. The stream processing approach, e.g. calculating the moving average of the twenty latest sensor values, is a good fit for this kind of analysis.

A combination of stream processing (for detecting current anomalous situations) and batch processing (for historical analysis) is therefore needed for a multi-purpose sensor data analysis platform. This paper describes the analysis cloud, a platform for the reliable execution of large numbers of small scale statistical analysis programs for sensor data. The platform supports both batch processing and stream processing.

\section{RELATED WORK}

\subsection{Batch Processing}

Hadoop (White, 2009) (Hadoop Website, 2012) is one of the most well-known batch processing systems currently in use. It is a framework for distributed processing of large data sets across a cluster of machines. The design and implementation is inspired by the papers on MapReduce (Dean and Ghemawat, 2004) and the Google File System (GFS) (Ghemawat et al., 2003). At its core, Hadoop consists of the MapReduce engine and the Hadoop Distributed File System (HDFS).
The file system ensures that the data is stored reliably on the nodes in the cluster. The engine allows applications to be split up into many small fragments of work. These fragments are executed on nodes in the cluster in such a way that the input data is close in terms of latency.

Disco (Disco Website, 2012) is a large scale data analysis platform. Its goals and design are very similar to those of Hadoop. Disco also provides a MapReduce engine and the Disco Distributed File System (DDFS). The main difference lies in the chosen programming languages. The MapReduce engine of Disco is written in Erlang, which is a language designed for building robust, fault-tolerant, distributed applications. The user applications themselves are written in Python.

Spark (Zaharia et al., 2010) (Spark Website, 2012) is a cluster computing system that aims to make data analysis fast. It provides primitives for in-memory cluster computing, so that repeated access to data is much quicker than with disk-based systems like Hadoop and Disco. Although Spark is a relatively new system, it can access any data source supported by Hadoop, making it easy to run over existing data.

Akka (Munish, 2012) (Akka Website, 2012) is a toolkit for building distributed, fault tolerant, eventdriven applications on the Java Virtual Machine (JVM). Akka uses actors, lightweight concurrent entities, to asynchronously process messages. This raise in abstraction level relieves developers from low-level issues in distributed systems, such as threads and locks. Actors are location transparent by design, which means that the distribution of an application is not hardcoded, but can be configured based on a certain topology at runtime.

Hadoop, Disco and Spark can all be used for historical sensor data analysis. However, because of their batch processing nature, it is awkward or even impossible to use them for detecting current anomalous situations. Akka is a toolkit for developing distributed applications and a lot of framework functionality is therefore still missing. Akka also does not guarantee message arrival, which makes it less suitable for data analysis.

\subsection{Stream Processing}

Esper (Esper Website, 2012) is a software component for processing large volumes of incoming messages or events. It is not an application or framework of itself, but can be plugged into an existing Java application. Esper is a Complex Event Processing (CEP) engine with its own domain specific language, called EPL, for processing events. EPL is a declarative 
language for dealing with high frequency time-based event data. It can be seen as an extension of SQL with support for time, causality and pattern matching.

S4 (Neumeyer et al., 2010) (S4 Website, 2012) is a distributed, scalable platform that allows programmers to develop applications for processing continuous unbounded streams of data. It does not have a distributed file system such as HDFS or DDFS, but relies on its own adapters to pull in data from external sources. S4 is not a CEP engine like Esper, because it does not have provisions to match patterns.

Storm (Storm Website, 2012) is comparable to S4 as it is also a scalable platform for processing continuous unbound streams of data. While S4 targets the Java language, Storm is designed to be used with any language that supports the Thrift interface. There is no internal distributed file system, instead Storm relies on integration with existing data sources such as databases and queuing systems such as Java Message Service (JMS).

Because Esper is a software component that can be plugged into an existing application, there is a lot of framework functionality still missing before it can be used to execute data analysis programs in a distributed and scalable way. Both S4 and Storm are platforms capable of running data analysis programs to detect current anomalous situations, and they can also be used for the analysis of historical sensor data. However, both lack specific support for sensor data analysis. The notion of simulated time, i.e. time that differs from the actual, current time, and the ability to flexibly schedule the analysis programs is missing.

\section{SENSOR DATA ANALYSIS REQUIREMENTS}

\subsection{Scalability and Reliability}

The analysis cloud is designed to execute analysis programs on a large number of computing nodes. The probability that one or more of these nodes fail increases with the number of nodes, so the analysis cloud should assume that computing nodes sometimes fail and handle this in a graceful way. If, for example, one node in a cluster of five nodes fails to perform its task, we expect the system to run at lower capacity, e.g. 80 percent, but not fail altogether. In other words, there should not be a critical single point of failure in the system. It is, however, acceptable if a non-critical function of the system becomes unavailable when a single component or node fails. Restarting the failed component or node should then restore that functionality without significant negative impact on the rest of the system.

In addition, adding nodes to the system should result in (near-)linear improvement in the overall capacity. For example, doubling the number of computing nodes in the system should result in (almost) twice the capacity. This horizontal scalability allows the manager of the system to add as many nodes to the system as needed to reach the desired overall performance.

\subsection{Modules}

The main purpose of the analysis cloud is the execution of analysis programs. It should be easy for developers to create these software modules. The interface that the module must adhere to should therefore contain as few methods as possible and each method should have a clear meaning.

The smallest conceivable interface would contain only a single callback function in the module, i.e. an execution function which is repeatedly called. However, this could result in a bad performance as the module is forced to create and destroy any connections to external data sources in this single function. To avoid such a performance penalty, the interface should also contain an initialization and termination callback function.

The creation of the module may depend on several parameters, such as definitions for the input and output sensors, or connectivity settings for external data sources. It should be left to the developer to construct and provision these parameters to the module as needed, based on a list of parameters supplied by the platform.

\subsection{Timers and Schedules}

Each module in the analysis cloud is given a timestamp to act upon in the execute callback function. This allows the module to select a specific sensor value from its external data source. To facilitate both batch and stream processing of data, it is necessary to have a notion of time that may differ from the actual, current time.

In the case of stream processing, each timestamp presented to the module may be equal to the current time. Shortly after the sensor data is stored in an external data source, the module asks for the data and processes it. In this case the analysis cloud is acting as an event processing engine.

In the case of batch processing, however, time should run from a moment in the past to a newer moment in time, likely also in the past. For example, in the case of the retailer running their sales performance 
batch, the time would run from Monday 0:00 hours to Sunday 23:59, while the run itself may be performed at any time after that.

Besides a live timer, it is therefore required to have the concept of a simulation timer, containing a time that is different from the actual time. It should also have an associated speed, which is a factor relative to the actual time. This is needed to pause the execution of a batch process (value equal to 0 ) or make the process go faster than the actual time window of the sensor data (value greater than 1).

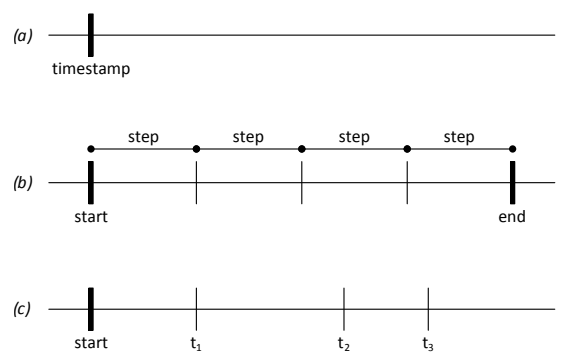

Figure 2: Different kinds of schedules: (a) Single schedule (b) Recurring schedule (c) Module driven schedule.

The analysis cloud should support different kinds of schedules. In the case of the retailer, the batch should run from a specific start time to a specific end time. This recurring schedule should also contain a step size, which denotes how much time there is between consecutive timestamps. The analysis cloud should also have the notion of a single schedule, which executes a module only for a single timestamp. Finally, it should be possible to have a schedule that depends on the sensor data at hand, e.g. to schedule based on the sample frequency. This leads to the need for a module driven schedule in which it is left to the module to decide what the next timestamp should be. See Figure 2 for a comparison of the different kinds of schedules.

\section{ARCHITECTURE OF THE ANALYSIS CLOUD}

The requirements listed in the previous chapter can be fulfilled with the architecture shown in Figure 3. Each solid block is a node in the system, either a physical machine or a virtual machine. The dotted lines denote a cluster of nodes containing the same kind of functionality. Sections 4.1 to 4.5 describe each component in more detail and section 4.6 shows how the whole system fulfills the mentioned requirements.

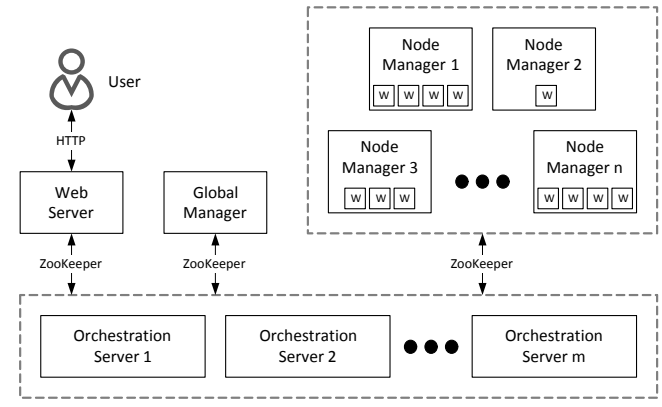

Figure 3: Architecture of the Analysis Cloud.

\subsection{Tasks}

There are multiple ways to link timers, schedules and modules. At least one timer is always available (the live timer) and there are possibly several simulation timers. The number of schedules is typically in the order of dozens, i.e. a bit higher than the number of timers. Finally, the number of modules is anywhere from a handful to a few thousand. Figure 4 shows an example of two timers, three schedules and four modules and their relationships.

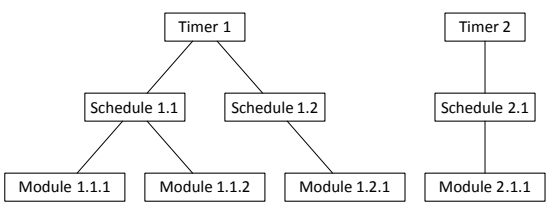

Figure 4: Hierarchy of Timers, Schedules and Modules.

The nodes executing the modules need to know in which order and from which timestamp each module should be executed. To keep track of this, there is the notion of tasks and a work queue containing these tasks. When a module enters the system, its first execution time is noted and the combination of this timestamp and a reference to the module is placed in the work queue. The tasks in the work queue are sorted by timestamp which means that the system can sleep until the timestamp at the front of the work queue has passed. The corresponding module is then executed and the new timestamp (if any) is determined, based on the module's linked schedule and timer.

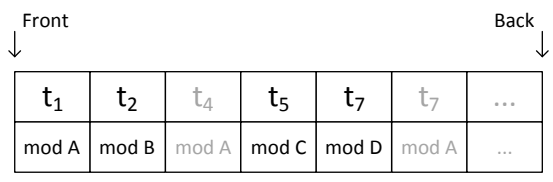

Figure 5: Work queue containing Tasks.

See Figure 5 for an example of a work queue containing tasks. Each black task is currently present, every grey one will be added as soon as its black coun- 
terpart has finished. In this example, the task for module $\mathrm{A}$ at timestamp $\mathrm{t}_{4}$ is added as soon as the execution of the module at timestamp $t_{1}$ has finished. The same applies to $t_{7}$ in combination with $t_{4}$ for the same module.

\subsection{Managers and Workers}

A node manager is a necessary part of every computing node. Each node manager supervises a part of the work of the total analysis cloud. The actual execution of the modules is performed by workers. Each worker listens to the work queue of the node manager and fetches a task as soon as one becomes available. Using multiple workers can speed up the processing when there is more than one processor core available or one analysis module is fetching data while another is using the processor. Typically there are two or more node managers present in the system, and one or more workers per node manager.

A node manager has two main functions. Its first function is supervising the execution of user modules. It uses its work queue containing tasks to decide which modules should be executed at what time. Its second function is to listen for additions, deletions and changes in the desired configuration of timers, schedules and modules. Based on this information, it makes changes in its internal configuration and reports on its current configuration.

There is a single global manager present in the system, which coordinates the work among all node managers. It listens to requests for changes in the total configuration of the system, i.e. the timers, schedules and modules. Based on this information it distributes the work among the node managers. It also listens to the current configuration of each node manager and reports on the combined configuration.

\subsection{Orchestration}

Except for the communication between the user and the web server, all communications between components of the analysis cloud are performed through an orchestration system. The ZooKeeper distributed coordination service (Hunt et al., 2010) (ZooKeeper Website, 2012) is used for the implementation of this part of the analysis cloud.

Figure 6 shows how the orchestration works for the communication of configuration data inside the system. Starting at the top, the user sets a desired global configuration, which is a combination of information about timers, schedules and modules. This information is automatically picked up by the global manager, which translates the global desired config-

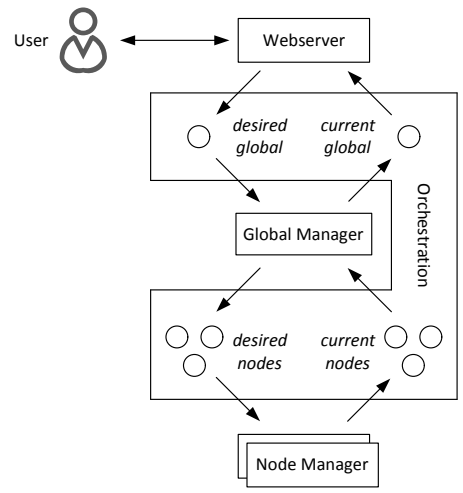

Figure 6: Orchestration. Each circle is a key/value pair containing configuration information.

uration into smaller parts, one for each node manager. The node managers automatically pick this up and use the configuration data to change their internal state. This state is then set in the current configuration of each node manager. The global manager automatically picks up these configurations and translates them into a global current configuration. The result of this is presented to the user.

\subsection{Web Interfaces}

There are two web interfaces present in the platform. One is a RESTful (Fielding, 2000) interface that uses the orchestration system to set the desired configuration of the analysis cloud and read its current configuration. It contains methods for listing all timers, schedules and modules, and methods to create and remove individual instances of those.

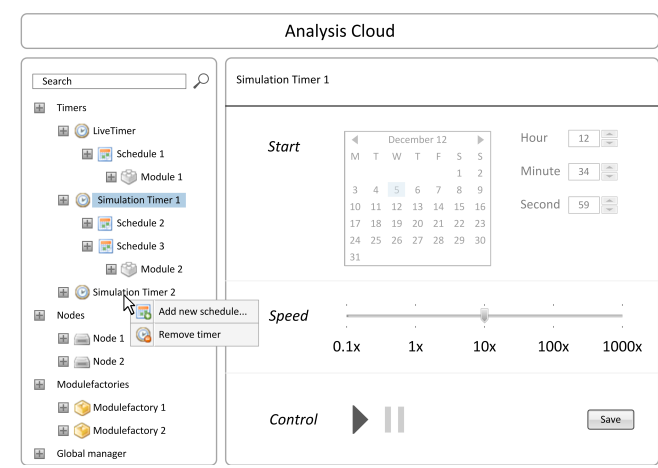

Figure 7: Website intended for the users of the Analysis Cloud and the developers of analysis modules.

The other interface is a set of web pages intended for users of the analysis cloud and the developers of analysis modules. Figure 7 shows the design of the website. The current hierarchy of timers, schedules and modules is shown on the left side of the screen, while the right side shows information on the cur- 
rently selected item. At the moment, the right mouse key is pressed and a pop-up menu is shown to add a schedule to the simulation timer or remove the timer altogether.

\subsection{Programming Interfaces}

The analysis cloud and its modules are written in Java. The developer of a module must implement a factory that is able to create a module with supplied parameters. The mandatory interface for factories looks like this:

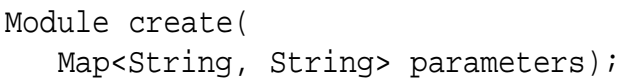

When the create function is called, the factory must create a new module and configure it with the supplied parameters, e.g. by using setters on the module.

Modules must also adhere to a specific interface to be used on the analysis cloud:

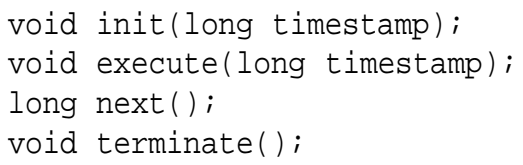

The init function is called just after the module has been constructed by the factory. The timestamp parameter tells the module which timestamp will be used for the first execution. This helps the module in its initialization, e.g. by being able to pre load data from an external source. The execute function is called one or more times after that for ascending timestamps. If the module driven schedule is used, the $n e x t$ function is called after each execution, to ask the module at which timestamp it would like to be scheduled again. Finally, the terminate function is called when the module is no longer needed, e.g. when the schedule has finished or the user deletes the module from the system.

Table 1: Callback functions with the number of calls to each function for the different types of schedule.

\begin{tabular}{l|l|l|l} 
& $\begin{array}{l}\text { Single } \\
\text { Sched- } \\
\text { ule }\end{array}$ & $\begin{array}{l}\text { Recur- } \\
\text { ring } \\
\text { Sched- } \\
\text { ule }\end{array}$ & $\begin{array}{l}\text { Module } \\
\text { Driven } \\
\text { Sched- } \\
\text { ule }\end{array}$ \\
\hline init & 1 & 1 & 1 \\
execute & 1 & $\mathrm{~s}^{a}$ & $\mathrm{~m}^{b}$ \\
next & 0 & 0 & $\mathrm{~m}^{b}$ \\
terminate & 1 & 1 & 0 or $1^{c}$
\end{tabular}

\footnotetext{
${ }^{a}$ depends on schedule: $\left\lfloor\frac{\text { end }- \text { start }}{\text { step }}+1\right.$

${ }^{b}$ depends on module: $[0, \infty]$

${ }^{c}$ depends on module
}

Table 1 lists the four callback functions of a module and shows how many times each function will be called for each type of schedule.

\subsection{Fulfillment of Requirements}

Section 3.1 states that there should not be a critical single point of failure in the system. Iterating through the components of the analysis cloud shows that this requirement is fulfilled by the following system features:

- If an orchestration server fails, the clients automatically connect to one of the remaining servers. Since all orchestration servers contain the same configuration data, there is no loss in functionality.

- If one of the node managers fails, the global manager will redistribute the work among the remaining node managers. A part of the work that the failed node manager has performed may be performed again by the node manager that took over.

- If the web server fails, the user is unable to monitor and control the analysis cloud. However, the node managers will continue with the currently configured work.

- If the global manager fails, the node managers still continue with the work they were given. Any updates the user provided on the website are postponed until the global manager is restarted.

Section 3.2 states that it should be easy for developers to create modules for the analysis cloud. Only two interfaces are mandatory, one for the factory and one for the module. The factory interface contains only one function and the module interface contains four functions, a compromise between ease of implementation and performance.

Finally, section 3.3 shows that it is necessary to have a notion of time that may differ from the actual, current time. For this purpose, the analysis cloud provides zero or more user-defined simulation timers with their own independent time and speed. The platform also provides three types of schedules to support multiple use cases.

\section{EXPERIMENTS ON THE ANALYSIS CLOUD}

Two experiments are performed to show how the analysis cloud behaves in practice. The first experiment, described in Section 5.1, shows that the platform is capable of running multiple modules with the same 
schedule. Modules started at a later time gradually catch up with the modules that were started earlier, without overloading the system. The second experiment, described in Section 5.2, shows that the platform is capable of distributing its workload over multiple computing nodes. Adding nodes to the system results in a shift of some tasks from the old nodes to the new ones.

\subsection{Single Node}

An external data source serves sensor data through a web interface. A single module fetches data from this web interface in its execute function and performs various calculations on the data, taking roughly 150 to 200 milliseconds. A recurring schedule is used with a step size of one second and a simulation timer with normal speed.

At the start of the experiment, a single module is created. Two minutes later, a second module is started with the same schedule and four minutes from the start a third module is started, also with the same schedule. Each module uses its own set of input and output sensor values. A single node manager is active during this experiment to be sure that all module executions take place on the same machine.

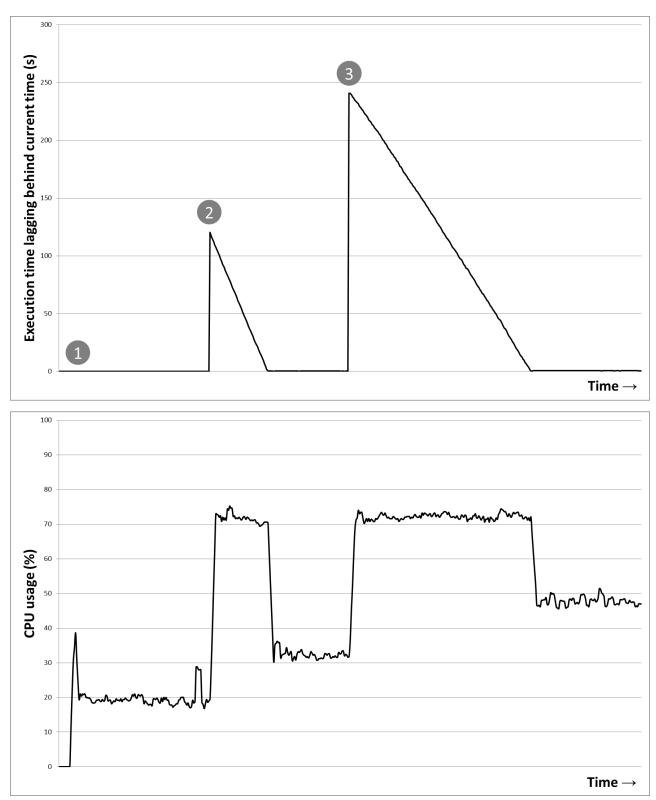

Figure 8: Result of the single node experiment. The top chart shows the number of seconds the timestamp for the module execution lags behind the actual time. The bottom chart shows the corresponding CPU usage.

Figure 8 shows the results of the experiment. The first module is started at moment 1 . A small peak in the CPU load is seen, followed by a steady line at about 20 percent.
The second module is started at moment 2 . The schedule and its timer were started when the first module was created, which means that the current time is now two minutes (120 seconds) ahead of the start time of the schedule and the module is executed repeatedly to catch up. This leads to a CPU usage of about 75 percent until the time gap is gone and the CPU usage drops to about 35 percent.

The third module is started at moment 3 . The gap between the current time and the timestamp used for the execution of the module is now four minutes ( 240 seconds). Catching up now takes even more time, not only because of this larger gap, but also because the system now runs three modules instead of two. This again leads to a CPU usage of about 75 percent until the time gap is gone and the CPU usage drops to about 50 percent.

\subsection{Multiple Nodes}

Multiple computing nodes are active in the analysis cloud during this experiment. At the start of the experiment, a large number of modules is configured and a single node is started. Two minutes later a second node is started, four minutes later a third, and six minutes later a fourth.

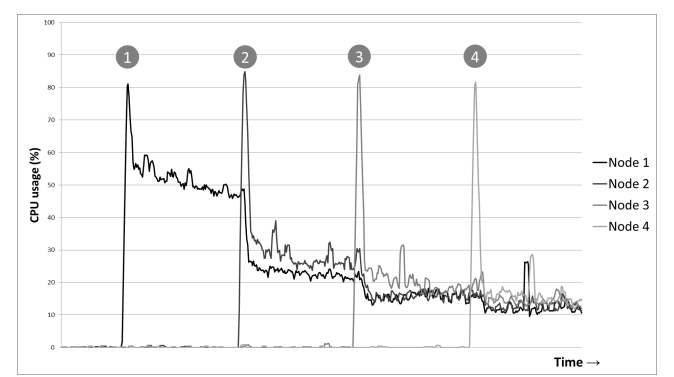

Figure 9: Result of the multiple nodes experiment. The chart shows the CPU usage over time as nodes are added to the analysis cloud.

Figure 9 shows the results of the experiment. The first node is started at moment 1. A peak in the CPU usage is seen, followed by a fairly steady line of about 50 percent. The CPU usage of the other (inactive) nodes is then still about 0 percent.

The second node is started at moment 2. At that moment the global manager redistributes the modules among both node managers, which results in a lower CPU usage of node 1. The CPU usage of node 2 is roughly the same, about 25 percent. The same applies to moments 3 and 4 where a third and a fourth node are started. The CPU usage of all four nodes is then roughly the same at about 15 percent. 


\section{CONCLUSIONS}

Several platforms and frameworks exist for the analysis of data. However, none of the currently existing solutions is tailored for the needs of sensor data analysis.

This paper presents a system that supports the processing of both live sensor data feeds and batches of historical sensor data. It contains simulation timers and different schedules including single, recurring and module driven. Moreover, the analysis cloud has simple programming interfaces, which makes it easy to develop analysis modules.

Experiments demonstrate that the system is capable of running analysis modules in a robust manner, and can catch up quickly when there is a discrepancy between the timestamp for an execution of a module and the actual time. Also, the capacity of the analysis cloud can be scaled up or down on demand by adding or removing computing nodes from the system.

The current analysis cloud leaves the actual retrieval and storage of sensor data to the module, i.e. each module must communicate with an external storage system before and after it performs calculations on the sensor data. It is therefore possible that multiple modules extract the same data from the storage system. In a future version of the analysis cloud, we would like to avoid the cost of this redundant retrieval.

The experiments in this paper are limited in scale and time. Using the analysis cloud for a longer period of time, with a larger number of nodes, will result in better understanding of its features and weaknesses. In future work we would like to assess this usage to better answer questions about scalability and limiting factors.

\section{ACKNOWLEDGEMENTS}

This publication is supported by the Dutch national programs Flood Control 2015 and COMMIT.

\section{REFERENCES}

Akka Website (2012). Akka toolkit for event-driven applications on the jvm. http://akka.io.

Blackwell, W. (2005). A neural-network technique for the retrieval of atmospheric temperature and moisture profiles from high spectral resolution sounding data. IEEE Transactions on Geoscience and Remote Sensing.

Dean, J. and Ghemawat, S. (2004). Mapreduce: Simplified data processing on large clusters. Symposium on Operating Systems Design and Implementation.
Disco Website (2012). Disco distributed computing framework. http://discoproject.org.

Esper Website (2012). Esper complex event processing. http: //esper.codehaus.org.

Fielding, R. T. (2000). Architectural styles and the design of network-based software architectures. http://www.ics.uci.edu/ fielding/pubs/ dissertation/fielding_dissertation.pdf.

Ghemawat, S., Gobioff, H., and Leung, S.-T. (2003). The google file system. ACM Symposium on Operating Systems Principles.

Hadoop Website (2012). Apache hadoop. http: / / hadoop. apache.org.

Hunt, P., Konar, M., Junqueira, F. P., and Reed, B. (2010). Zookeeper: Wait-free coordination for internet-scale systems. USENIX Annual Technical Conference.

IBM Corporation (2001). Autonomic computing: Ibms perspective on the state of information technology. http://www.research.ibm.com/autonomic/ manifesto/autonomic_computing.pdf.

Munish, K. G. (2012). Akka Essentials. Packt Publishing.

Neumeyer, L., Robbins, B., Nair, A., and Kesari, A. (2010). S4: Distributed stream computing platform. IEEE International Conference on Data Mining Workshops.

Pyayt, A., Mokhov, I., Lang, B., Krzhizhanovskaya, V., and Meijer, R. (2011). Machine learning methods for environmental monitoring and flood protection. International Conference on Artificial Intelligence and Neural Networks.

Rao, J., Bu, X., Xu, C.-Z., and Wang, K. (2011). A distributed self-learning approach for elastic provisioning of virtualized cloud resources. IEEE International Symposium on Modelling, Analysis, and Simulation of Computer and Telecommunication Systems.

S4 Website (2012). S4 distributed stream computing platform. http://incubator. apache.org/s4.

Sheng, B., Li, Q., and Mao, W. (2006). Data storage placement in sensor networks. ACM International Symposium On Mobile Ad Hoc Networking and Computing.

Spark Website (2012). Spark cluster computing framework. http: //www. spark-project.org.

Storm Website (2012). Storm distributed realtime computation system. http://storm-project. net.

White, T. (2009). Hadoop: The Definitive Guide. O'Reilly Media.

Zaharia, M., Chowdhury, M., Franklin, M. J., Shenker, S., and Stoica, I. (2010). Spark: Cluster computing with working sets. HotCloud.

ZooKeeper Website (2012). Apache zookeeper. http:// zookeeper.apache.org. 